\title{
A water wave mixed type problem: existence of periodic travelling waves for a 2D Boussinesq system
}

\author{
José R. Q uintero* \\ Departamento de Matemáticas, Universidad del Valle, Cali, Colombia
}

\begin{abstract}
In this paper we establish the existence of periodic travelling waves for a 2D Boussinesq type system in threedimensional water-wave dynamics in the weakly nonlinear long-wave regime. For wave speed $|c|>1$ and large surface tension, we are able to characterize these solutions through spatial dynamics by reducing a linearly ill-posed mixed type initial value problem to a center manifold of finite dimension and infinite codimension. We will see that this center manifold contains all globally defined small-amplitude solutions of the travelling wave equation for the Boussinesq system that are periodic in the direction of propagation.
\end{abstract}

Key words: periodic travelling waves, center manifold approach, stability

Un problema de ondas de agua de tipo mixto: la existencia de ondas viajeras periódicas para un sistema Boussinesq 2D

\section{Resumen}

En este artículo establecemos un resultado de existencia de ondas periódicas para un sistema $2 \mathrm{D}$ del tipo Boussinesq que describe la evolución de ondas de agua de gran elongación y pequeña ampitud que son débilmente lineales. Para velocidad de onda $|c|>1$ y tensión superficial suficientemente grande, estas soluciones son caracterizadas a través de dinámica espacial al reducir el problema de valor inicial (mal puesto a nivel lineal) a una variedad central de dimensión finita y codimensión infinita. Se mostrará que dicha variedad central contiene todas las soluciones de onda viajera de pequenã amplitud para el sistema tipo Boussinesq que son periódicas en la dirección de propagación de la onda.

Pal abras clave: ondas viajeras periódicas, método de variedad central, estabilidad.

\section{Introduction}

This paper presents an existence result of nonlinear travelling water waves which are periodic in their direction of propagation and have a pulse structure in the unbounded transverse spatial direction. As done for a wide range of applications, we use spatial dynamics and center manifold reduction to obtain such a result in a model related with the water-wave problem. Here the expression "spatial dynamics" means to perform a method in which a system of partial differential equations governing a physical problem is formulated as a evolutionary equation (in general ill-posed)

$$
u_{\zeta}=A(u)+G(u) .
$$

in which an unbounded spatial coordinate plays the role of the timelike variable $\zeta$ (see Kirchgässner (1982)). In this paper the method is applied to studying the existence of non trivial periodic travelling wave for the Boussinesq type system related with the water wave problem

$$
\left\{\begin{array}{r}
\eta_{t}+\epsilon \nabla \cdot\left(\eta\left(\Phi_{x}^{p}, \Phi_{y}^{p}\right)\right)+\Delta \Phi-\frac{\mu}{6} \Delta^{2} \Phi=0 \\
\Phi_{t}+\eta-\mu\left(\sigma-\frac{1}{2}\right) \Delta \eta+\frac{\epsilon}{p+1}\left(\Phi_{x}^{p+1}+\Phi_{y}^{p+1}\right)=0
\end{array}\right.
$$

where $\sqrt{\mu}=h_{0} / L$ is the ratio of undisturbed fluid depth to typical wave length (long-wave parameter or disper-

*Correspondencia: J. R. Quintero: jose.quintero@correounivalle.edu.co. Recibido marzo 2014; aceptado diciembre 2014. 
sion coefficient), and $\epsilon$ is the ratio of typical wave amplitude to fluid depth (amplitude parameter or nonlinearity coefficient), $\sigma^{-1}$ is the Bond number (associated with the surface tension), $\Phi$ is the rescale nondimensional velocity potential on the bottom $z=0$, and $\eta$ is the rescaled free surface el evation. We consi der waves which are periodic in a moving frame of reference, so that they are periodic in the variable $x-c t$, where $t$ denotes the temporal variable. For this physical problem, we have a bounded spacelike coordinates (the vertical direction), which is bounded because the fluid has finite depth, and the coordinate $x-c t$ which is considered bounded be cause we are looking for periodic wave in this variable. So, since there is not any restriction upon the behavior of the waves in the spatial direction $y$ transverse to their direction of propagation, we are allowed to use it as timelike variable. We apply spatial dynamics to the problem by formulating this as an evolutionary system of the form (1.1) with $\zeta=y$ in an infinite-dimensional phase space consisting of periodic functions of $x-c t$ (see G rover \& Schneider (2001), Sandstede \& Scheel (1999), Sandstede \& Scheel (2004), and Haragus-Courcelle \& Schneider (1999) for applications to respectively nonlinear wave equations, reaction-diffusion equations, and Taylor-Couette problems, and Quintero \& Pego (2002) for periodic nonlinear travelling for the Benney-Luke model).

In the case of wave speed $|c|>1$ and bond number $\sigma>\frac{1}{2}$, the Boussinesq system (1.2) under consideration in this paper has a very close relationship with the Benney-Luke model derived in Q uintero \& Pego (1999) when $a>b$ and also with the KP-Il model $\left(\sigma>\frac{1}{3}\right)$, in the sense that travelling waves for the Boussinesq system (1.2) can generate travelling waves for the Benney-Luke model and the KP-II model, up to some order. Event tough this fact, in the regime of wave speed $|c|>1$ and bond number $\sigma>\frac{1}{2}$, the Boussinesq system (1.2) seems to be closer to the linearized system of the exact water wave problem with surface tension (see G rover (2001), G rover \& M ielke (2001)) since both models share ill-posed spatial evolution equations with finite-dimensional center manifolds, while for the Benney-Luke model in the same regime and also for the KP-II model there are ill-posed spatial evolution equations with infinite-dimensional center manifolds.

It is well known that a finite-dimensional dynamical system whose linear part has purely imaginary eigenvalues admits an invariant manifold called the center manifold which contains all its small, bounded solutions. The dimension of the center manifold is determined by the number of purely imaginary eigenvalues (e.g., see Vanderbauwhede (1989)). This type of results under special hypotheses has been extended for infinite-dimensional evolutionary systems whose linear part has either finite or even infinite number of purely imaginary eigenvalues, showing in particular that the original problem is locally equivalent to a system of ordinary differential equations whose solutions can be expressed in terms of the solution on the center space (tangent to the center manifold). A generalization regarding invariant manifolds of infinite dimension and codimension in nonautonomous systems was obtained by Scarpellini (1990), but his hypotheses require that the operator $A_{1}$ ( $A$ restricted to the hyperbolic space) be bounded from $H$ to $H$. We also have some works by M ielke (1991) and Vanderbauwhede \& looss (1982), Q uintero \& Pego (2002), among others. The general strategy of our proof will follow closely the lines of Vanderbauwhede (1989) (also see Q uintero \& Pego (2002), Kirchgässner (1982), Mielke (1988), M ielke (1992) for the case of a finite-dimensional center manifold in an ill-posed system for which the spectrum of $A_{1}$ is unbounded on both sides of the imaginary axis. In order to accomplish this, we need to transform the travelling wave system into an integral equation that must contain all small bounded solutions. By modifying the nonlinearity $f$ outside a neighborhood of 0 using a cutoff function, we are able to obtain an invariant local manifold as a consequence of the contraction mapping argument.

The main differences between our results and those in Vanderbauwhede \& looss (1982) are that our cutoff occurs in a space with less regularity than the space regularity and also that the nonlinear part has a smoothing property. This facts are clever to control the norm of solutions in the right space using an indefinite energy which turns out to be "definite" on the center manifold. The stronger assumption on the nonlinear term $f$ imposed in the present work is completely natural for the present application to the Boussinesq system.

In this paper we describe all small travelling waves that translate steadily for $\sigma>\frac{1}{2}$ with supercritical speed $|c|>1$, which are periodic in the direction of translation (or orthogonal to it). In this regime, after rescaling $\epsilon$ and $\mu$, the traveling-wave system for (1.2) takes the form

$$
\left(\begin{array}{c}
\Delta v-\frac{1}{6} \Delta^{2} v+\nabla \cdot\left(u\left(v_{x}^{p}, v_{y}^{p}\right)\right)-c u_{x} \\
u-\left(\sigma-\frac{1}{2}\right) \Delta u+\frac{1}{p+1}\left(v_{x}^{p+1}+v_{y}^{p+1}\right)-c v_{x}
\end{array}\right)=\left(\begin{array}{l}
0 \\
0
\end{array}\right) .
$$

Existence of periodic travelling waves follows by considering the system (1.3) as an evolution equation where $y$ acts as the "time" variable. In this case when we seek for $x$-periodic travelling wave solutions, the initial-value problem for the system (1.3) considered as an evolution equation in the variable $y$ has mixed type due to the fact that the Cauchy problem turns out to be linearly ill-posed for wave speed $|c|$ large enough and large bond number 
$\left(\sigma>\frac{1}{2}\right)$. To be more precise, at the linear level there are finite many central modes (pure imaginary eigenvalues) and infinitely many hyperbolic modes. As a consequence of this fact, the existence result of $x$-periodic travelling wave solutions involves using an invariant center manifold of finite dimension and infinite codimension. This center manifold contains all globally defined smallamplitude solutions of the travelling wave equation for the Boussinesq system that are $x$-periodic in the direction of propagation.

\section{Periodic travelling waves for $|c|>1$ and $\sigma>\frac{1}{2}$}

Recall that $x$-periodic travelling-wave profile $(u, v)$ should satisfy the system (1.3). In order to look for the existence of $x$-periodic travelling waves of period $2 \pi$, we set the new variables

$$
\begin{array}{r}
u_{1}=\partial_{x} v, \quad u_{2}=\partial_{y} v, \\
u_{3}=\partial_{y y} v, \quad u_{4}=\partial_{y y y} v, \\
u_{5}=u, \quad u_{6}=\partial_{y} u,
\end{array}
$$

then we have for $\gamma\left(\sigma-\frac{1}{2}\right)=1$ that

$$
\begin{gathered}
\partial_{y} u_{1}=\partial_{x} u_{2}, \partial_{y} u_{2}=u_{3}, \partial_{y} u_{2}=u_{4} \\
\partial_{y} u_{4}=6 \partial_{x} u_{1} \partial_{x}^{3} u_{1}+6 u_{3}-2 \partial_{x}^{2} u_{3}-6 \partial_{x} u_{5}+ \\
6 \partial_{x}\left(u_{5} u_{1}^{p}\right)+6 u_{6} u_{2}^{p}+6 p u_{5} u_{2}^{p-1} u_{3} \\
\partial_{y} u_{5}=u_{6} \\
\partial_{y} u_{6}=-c \gamma u_{1}+\gamma u_{5}-\partial_{x}^{2} u_{5}+ \\
+\frac{\gamma}{p+1}\left(u_{1}^{p+1}+u_{2}^{p+1}\right)
\end{gathered}
$$

In terms of the new variable $U^{t}=\left(u_{1}, u_{2}, u_{3}, u_{4}, u_{5}, u_{6}\right)$, we see that this system can be rewritten as an evolution in which $y$ is considered as the time variable

$$
\partial_{y} U=A U+G(U),
$$

where we have that

$$
\mathcal{A}=\left(\begin{array}{cccccc}
0 & \partial_{x} & 0 & 0 & 0 & 0 \\
0 & 0 & I & 0 & 0 & 0 \\
0 & 0 & 0 & I & 0 & 0 \\
6 \partial_{x}-\partial_{x}^{3} & 0 & 6 I-\partial_{x}^{2} & 0 & -6 c \partial_{x} & 0 \\
0 & 0 & 0 & 0 & 0 & I \\
-c \gamma & 0 & 0 & 0 & \gamma-2 \partial_{x}^{2} & 0
\end{array}\right)
$$

and also

$$
G(U)=\left(\begin{array}{c}
0 \\
0 \\
0 \\
6 \partial_{x}\left(u_{5} u_{1}^{p}\right)+6 u_{6} u_{2}^{p}+6 p u_{5} u_{2}^{p-1} u_{3} \\
0 \\
\frac{\gamma}{p+1}\left(u_{1}^{p+1}+u_{2}^{p+1}\right)
\end{array}\right)
$$

Now, for a given integer $r \geq 0$, let $\tilde{H}^{r}$ denote the Sobolev space of $2 \pi$-periodic functions on $\mathbb{R}$ whose weak derivatives up to order $k$ are square-integrable. Then $\tilde{H}^{r}$ is a Hilbert space with norm given by

$$
\|u\|_{\tilde{H}^{k}}^{2}=\sum_{j=0}^{k} \int_{0}^{2 \pi}\left|\partial_{x}^{j} u\right|^{2} d x
$$

We will study the existence of $x$-periodic solutions for (2.8) in the Hilbert spaces $H$ and $X$ defined by

$$
\begin{aligned}
& H=\tilde{H}^{1} \times \tilde{H}^{1} \times \tilde{H}^{0} \times \tilde{H}^{-1}, \\
& X=\tilde{H}^{2} \times \tilde{H}^{2} \times \tilde{H}^{1} \times \tilde{H}^{0} .
\end{aligned}
$$

Note that $X$ is densely embedded in $H$. If we assume that $U(x ; y)=\sum_{n \in \mathbb{Z}} \widehat{U}(n, y) e^{i n x}$, then we see that

$$
\partial_{y} \widehat{U}(n)=\widehat{\mathcal{A}}(n) \widehat{U}(n, y)+\widehat{G}_{n}(U),
$$

where the matrix $\widehat{\mathcal{A}}(n)$ has the form

$$
\widehat{\mathcal{A}}(n)=\left(\begin{array}{cccccc}
0 & i n & 0 & 0 & 0 & 0 \\
0 & 0 & 1 & 0 & 0 & 0 \\
0 & 0 & 0 & 1 & 0 & 0 \\
6 i n+i n^{3} & 0 & 6+2 n^{2} & 0 & -6 i c n & 0 \\
0 & 0 & 0 & 0 & 0 & 1 \\
-c \gamma & 0 & 0 & 0 & \gamma+2 n^{2} & 0
\end{array}\right)
$$

It is straightforward to see that the characteristic polynomial $\mathcal{P}(n, \beta)$ of $\widehat{\mathcal{A}}(n)$ is given by

$$
\begin{aligned}
\mathcal{P}(n, \beta) & =\beta^{6}-\left(6+3 n^{2}+\gamma\right) \beta^{4}+\left[\left(6+2 n^{2}\right)\left(n^{2}+\gamma\right)\right. \\
& \left.+n^{2}\left(6+n^{2}\right)\right] \beta^{2}+n^{2}\left[6 \gamma c^{2}-\left(6+n^{2}\right)\left(n^{2}+\gamma\right)\right],
\end{aligned}
$$

where $\gamma>0,|c|>0$ and $n \in \mathbb{Z}$. We note that the eigenvalues for $\widehat{\mathcal{A}}(n)$ are roots of the cubic polynomial $p_{n}$ in the variable $\lambda=\beta^{2}$ given by

$$
p_{n}(\lambda)=\lambda^{3}+a_{2}(n) \lambda^{2}+a_{1}(n) \lambda+a_{0}(n),
$$

where $a_{0}(n), a_{1}(n)$ and $a_{2}(n)$ are defined as

$$
\begin{aligned}
a_{2}(n) & =-\left(3 n^{2}+6+\gamma\right) \\
a_{1}(n) & =\left(6+2 n^{2}\right)\left(n^{2}+\gamma\right)+n^{2}\left(6+n^{2}\right) \\
& =3 n^{4}+2(6+\gamma) n^{2}+6 \gamma \\
a_{0}(n) & =n^{2}\left[6 \gamma c^{2}-\left(6+n^{2}\right)\left(n^{2}+\gamma\right)\right] \\
& =-n^{2}\left[n^{4}+(6+\gamma) n^{2}+6 \gamma\left(1-c^{2}\right)\right]
\end{aligned}
$$




\section{Eigenvalues of $\widehat{\mathcal{A}}(n)$}

We want to compute the eigenvalues of $\widehat{\mathcal{A}}(n)$. For $n=0$, we have that $a_{0}(0)=0, a_{1}(0)=6 \gamma>0$, $a_{2}(0)=-(6+\gamma)<0$ and the cubic equation (2.12) can be easily solve since

$$
\begin{aligned}
\lambda^{3}-(6+\gamma) \lambda^{2}+6 \gamma \lambda & =\lambda\left(\lambda^{2}-(6+\gamma) \lambda+6 \gamma\right) \\
& =\lambda(\lambda-\gamma)(\lambda-6)=0 .
\end{aligned}
$$

In other words, we have the existence of six eigenvalues for $\widehat{\mathcal{A}}^{(0)}$ given by,

$$
\begin{aligned}
& \beta_{1}(0)=\beta_{2}(0)=0, \\
& \beta_{3}(0)=\sqrt{\gamma}=-\beta_{4}(0), \\
& \beta_{5}(0)=\sqrt{6}=-\beta_{6}(0) .
\end{aligned}
$$

Assume now that $n \neq 0$. We will see that for $\gamma>0$ and $c^{2}>1$ large enough, the polynomial $p_{n}$ has a real root $\lambda_{1}(n)$ which is negative for a finite number of $n$ 's and positive for infinitely many $n$ 's. To do this we need somehow to localize the roots of the polynomial $p_{n}^{\prime}$ for $n \neq 0$. Note that

$$
p_{n}^{\prime}(\lambda)=3 \lambda^{2}+2 a_{2}(n) \lambda+a_{1}(n)
$$

has real roots given by

$$
\begin{aligned}
& \rho_{+}(n)=-\frac{a_{2}(n)}{3}+\frac{\sqrt{a_{2}^{2}(n)-3 a_{1}(n)}}{3}, \\
& \rho_{-}(n)=-\frac{a_{2}(n)}{3}-\frac{\sqrt{a_{2}^{2}(n)-3 a_{1}(n)}}{3}
\end{aligned}
$$

since

$$
a_{2}^{2}(n)-3 a_{1}(n)=\gamma^{2}-6 \gamma+36=(\gamma-3)^{2}+27>0 .
$$

Moreover, a direct computation shows that

$$
\begin{array}{r}
p_{n}\left(\rho_{ \pm}\right)=\frac{2 a_{2}^{3}(n)}{27}-\frac{a_{1}(n) a_{2}(n)}{3}+a_{0}(n) \pm \frac{2}{27}\left(3 a_{1}(n)\right. \\
\left.-a_{2}^{2}(n)\right) \sqrt{a_{2}^{2}(n)-3 a_{1}(n)} \\
=\frac{2 a_{2}^{3}(n)}{27}-\frac{a_{1}(n) a_{2}(n)}{3}+a_{0}(n) \\
\mp \frac{2}{27}\left(\gamma^{2}-6 \gamma+36\right)^{\frac{3}{2}}
\end{array}
$$

From this, we conclude for any $n \neq 0$ that

$$
p_{n}\left(\rho_{-}\right)-p_{n}\left(\rho_{+}\right)=\frac{4}{27}\left(\gamma^{2}-6 \gamma+36\right)^{\frac{3}{2}}>0
$$

On the other hand, we have that

$$
\begin{aligned}
\frac{2 a_{2}^{3}(n)}{27} & -\frac{a_{1}(n) a_{2}(n)}{3}+a_{0}(n) \\
& =\frac{1}{27}\left(162 \gamma c^{2} n^{2}+54 \gamma(6+\gamma)-2(6+\gamma)^{3}\right) \\
& =\frac{1}{27}\left(162 \gamma c^{2} n^{2}-2(6+\gamma)(\gamma-3)(\gamma-12)\right) .
\end{aligned}
$$

Using this we have that

$$
\begin{aligned}
& p_{n}\left(\rho_{ \pm}\right)=\frac{1}{27}\left(162 \gamma c^{2} n^{2}-2(6+\gamma)(\gamma-3)(\gamma-12)\right. \\
& \left.\mp 2\left(\gamma^{2}-6 \gamma+36\right)^{\frac{3}{2}}\right)
\end{aligned}
$$
and that $f^{\prime}(0)=f^{\prime}(6)=0$, so we have that

$$
f(\gamma)=3^{5} \gamma^{2}\left(\gamma^{2}-6\right)^{2}>0
$$

which implies that

$$
\left(\gamma^{2}-6 \gamma+36\right)^{\frac{3}{2}}>(6+\gamma)(\gamma-3)(\gamma-12)
$$

and so, we have for $\gamma>0$ that

$$
\begin{aligned}
p_{n}\left(\rho_{-}\right) & =\frac{1}{27}\left(162 \gamma c^{2} n^{2}+2\left(\gamma^{2}-6 \gamma+36\right)^{\frac{3}{2}}\right. \\
& -2(6+\gamma)(\gamma-3)(\gamma-12))>\frac{162}{27} \gamma c^{2} n^{2}>0 .
\end{aligned}
$$

Now we note that

$$
\begin{gathered}
p_{n}\left(\rho_{+}\right)=\frac{1}{27}\left(162 \gamma c^{2} n^{2}-2((6+\gamma)(\gamma-3)(\gamma-12)\right. \\
\left.\left.+\left(\gamma^{2}-6 \gamma+36\right)^{\frac{3}{2}}\right)\right)
\end{gathered}
$$

so, we have that $p_{n}\left(\rho_{+}\right)$is positive for $|n|$ large and could be negative for a finite number of $n$ 's. As a consequence of this, for $n \neq 0$ the polynomial $p_{n}$ has a real root $\lambda_{1}(n)$, whose sign depends on the sign of $a_{0}(n)$. In the case $p_{n}\left(\rho_{+}\right)>0$, there are two conjugate complex roots $\lambda_{2}(n)$ and $\lambda_{3}(n)=\overline{\lambda_{2}(n)}$, and in the case $p_{n}\left(\rho_{+}\right)<0$ there are two positive real root $\lambda_{2}(n) \geq \lambda_{3}(n)>0$. Finally, we want to determine the sign of $\lambda_{1}(n)$ for $\gamma>0$. We first observe for $c^{2}>1$ large enough and $\gamma>0$ that there is $n_{0} \neq 0$ such that

$$
a_{0}(n)>0 \text { for } 0<n \leq n_{0} \text {, and } a_{0}(n)<0 \text { for } n>n_{0} \text {. }
$$

From this fact, we conclude for $c^{2}>1$ large enough and $\gamma>0$ that

$$
\lambda_{1}(n)<0 \text { for } 0<n \leq n_{0} \text {, and } \lambda_{1}(n)>0 \text { for } n>n_{0} .
$$




\section{Right and left eigenvectors of $\widehat{\mathcal{A}}(n)$}

As we established above, $\beta_{1}(0)=\beta_{2}(0)=0$ is an eigenvalue. We see directly that the eigenvectors are

$$
v_{1}(0)=(1,0,0,0, c, 0)^{t}, \quad v_{2}(0)=(0,1,0,0,0,0)^{t} .
$$

and left eigenvectors

$$
z_{1}(0)=(1,0,0,0, c, 0)^{t}, \quad z_{2}(0)=\left((0,1,0,-1 / 6,0,0)^{t} .\right.
$$

The eigenvalue $\beta_{3}(0)=\sqrt{\gamma}=-\beta_{4}(0)$ are single eigenvalues with right eigenvectors

$$
v_{3}(0)=(0,0,0,0,1, \sqrt{\gamma})^{t}, \quad v_{4}(0)=(0,0,0,0,1,-\sqrt{\gamma})^{t} .
$$

and left eigenvectors

$$
\begin{aligned}
& z_{3}(0)=\frac{1}{2 \sqrt{\gamma}}(c \sqrt{\gamma}, 0,0,0, \sqrt{\gamma}, 1)^{t}, \\
& z_{4}(0)=\frac{-1}{2 \sqrt{\gamma}}(c \sqrt{\gamma}, 0,0,0,-\sqrt{\gamma}, 1)^{t} .
\end{aligned}
$$

On the other hand, the eigenvalue $\beta_{5}(0)=\sqrt{6}=-\beta_{6}(0)$ are single eigenvalues with right eigenvectors

$$
v_{5}(0)=(0,1, \sqrt{6}, 0,1,0)^{t}, \quad v_{6}(0)=\left((0,1,-\sqrt{6}, 0,1,0)^{t} .\right.
$$

and left eigenvectors

$$
\begin{aligned}
& z_{5}(0)=\frac{1}{12}(0,0, \sqrt{6}, 1,0,0)^{t} \\
& z_{6}(0)=\frac{1}{12}(0,0,-\sqrt{6}, 1,0,0)^{t} .
\end{aligned}
$$

Now, we will describe the form of the eigenvalues for $\widehat{\mathcal{A}}(n)$ for $n \neq 0$. In this case, we have that

$$
\begin{aligned}
& \beta_{3}(n)=-\beta_{4}(n)=\sqrt{\lambda_{2}(n)} \in \mathbb{C}, \\
& \beta_{5}(n)=-\beta_{6}(n)=\sqrt{\lambda_{3}(n)} \in \mathbb{C}
\end{aligned}
$$

and for $\gamma>0$ we have that

$$
\begin{aligned}
& \beta_{1}(n)=-\beta_{2}(n)=\sqrt{\lambda_{1}(n)} \in i \mathbb{R}, 0 \leq|n| \leq n_{0}, \\
& \beta_{1}(n)=-\beta_{2}(n)=\sqrt{\lambda_{1}(n)} \in \mathbb{R},|n|>n_{0} .
\end{aligned}
$$

A direct computation shows for $1 \leq m \leq 6$ that $\beta_{m}(n)$ is a single eigenvalue with right eigenvector

$$
\begin{aligned}
v_{m}(n)=\left(i n, \beta_{m}(n), \beta_{m}^{2}(n),\right. & \beta_{m}^{3}(n), \\
& \left.-\frac{c n \gamma i}{\Theta_{m}(n)},-\frac{c n \gamma \beta_{m}(n) i}{\Theta_{m}(n)}\right)^{t},
\end{aligned}
$$

where $\Theta_{m}(n)=\beta_{m}^{2}(n)-\left(\gamma+n^{2}\right)$. It is also straightforward to show that left eigenvector $z_{m}(n)$ is given by

$$
\begin{aligned}
z_{m}(n)=Q(m, n)\left(\frac{q_{m}(n)}{i n \beta_{m}(n)},\right. & \frac{q_{m}(n)}{\beta_{m}^{2}(n)}, \beta_{m}(n), 1, \\
& \left.-\frac{c n \beta_{m}(n) i}{\Theta_{m}(n)},-\frac{6 c n i}{\Theta_{m}(n)}\right),
\end{aligned}
$$

where $q_{m}(n)=\beta_{m}^{2}(n)\left(\beta_{m}^{2}(n)-\left(6+n^{2}\right)\right)$ and $Q(m, n)$ is taken such that

$$
z_{m}(n) v_{l}(n)=\delta_{m}^{l} .
$$

If we introduce the matrices $Z(n)$ and $V(n)$ given by

$$
\begin{aligned}
& Z(n)=\left(\begin{array}{l}
z_{1}(n) \\
z_{2}(n) \\
z_{3}(n) \\
z_{4}(n) \\
z_{5}(n) \\
z_{6}(n)
\end{array}\right), \\
& V(n)=\left(v_{1}(n), v_{2}(n), v_{3}(n), v_{4}(n), v_{5}(n), v_{6}(n)\right),
\end{aligned}
$$

we have $Z(n) \cdot V(n)=I_{6}$ and

$$
\begin{aligned}
Z(n) \widehat{A}(k) V(k)=\operatorname{diag}( & \beta_{1}(n), \beta_{2}(n), \beta_{3}(n), \\
& \left.\beta_{4}(n), \beta_{5}(n), \beta_{6}(n)\right), n \in \mathbb{Z} .
\end{aligned}
$$

Now, we observe that given any vector $\widehat{U}(n) \in \mathbb{R}^{6}$, we may write

$$
\widehat{U}(n)=V(n) \cdot Z(n) \widehat{U}(n)=V(n) U^{\#}(n),
$$

where

$$
U^{\#}(n)=Z(n) \widehat{U}(n)=\left(\begin{array}{c}
U_{1}^{\#}(n) U_{2}^{\#}(n) \\
U_{3}^{\#}(n) \\
U_{4}^{\#}(n) \\
U_{5}^{\#}(n) \\
U_{6}^{\#}(n)
\end{array}\right)
$$

Using this representation, we have for $U=\sum_{n \in \mathbb{Z}} \widehat{U}(n) e^{i n x}$ that

$$
\left\{\begin{array}{l}
U=\sum_{n \in \mathbb{Z}} \sum_{m=1}^{6} v_{m}(n) U_{m}^{\#}(k) e^{i n x}, \\
\mathcal{A} U=\sum_{n \in \mathbb{Z}} \sum_{m=1}^{6} \beta_{m}(n) v_{m}(n) U_{m}^{\#}(n) e^{i k x} .
\end{array}\right.
$$

We define the projections $\pi_{0}$ and $\pi_{1}$ for $\gamma>0$ by

$$
\begin{aligned}
& \pi_{0} U= \sum_{0 \leq|n| \leq n_{0}} \sum_{m=1}^{2} v_{m}(n) U_{m}^{\#}(k) e^{i n x} \\
& \pi_{1} U=\sum_{0 \leq|n| \leq n_{0}} \sum_{m=3}^{6} v_{m}(n) U_{m}^{\#}(k) e^{i n x} \\
& \quad+\sum_{|n|>n_{0}} \sum_{m=1}^{6} v_{m}(n) U_{m}^{\#}(k) e^{i n x} .
\end{aligned}
$$


We see directly from the explicit form of the roots of the polynomial $p_{n}$ that $\left|\beta_{m}(n)\right|$ grows asymptotically linearly in $|n|$. In fact, we must recall that the roots $\lambda_{m}(n)$ of $\widehat{\mathcal{A}}(n)$ for $1 \leq m \leq 3$ depend on the discriminant $D(n)$ of $p_{n}$ defined as

$$
D(n)=Q^{3}(n)+R^{2}(n) .
$$

where $Q$ and $R$ are defined by

$$
\begin{aligned}
& Q(n)=\frac{3 a_{1}(n)-a_{2}^{2}(n)}{9} \\
& R(n)=\frac{9 a_{1}(n) a_{2}(n)-27 a_{0}(n)-2 a_{2}^{3}(n)}{54}
\end{aligned}
$$

More concretely, we havethefollowing known result that characterizes the roots for a cubic polynomial

1. if $D(n)$ is positive, then $p_{n}$ has one real root $\left(\lambda_{1}(n)\right)$ and two are complex conjugates $\left(\lambda_{2}(n)\right.$ and $\left.\lambda_{3}(n)=\overline{\lambda_{2}(n)}\right)$.

2. if $D(n)$ is negative, then $p_{n}$ has three different real roots, and

3. if $D(n)=0$, then $p_{n}$ has three real roots, with two of them are equal $\left(\lambda_{2}(n)=\lambda_{3}(n)\right)$.

Moreover, the roots of the polynomial $p_{n}$ defined in (2.12) are explicitly given by

$$
\begin{aligned}
& \lambda_{1}=-\frac{1}{3} a_{2}(n)+(S(n)+T(n)) \\
& \lambda_{2}=-\frac{1}{3} a_{2}(n)-\frac{1}{2}(S(n)+T(n))+\frac{i \sqrt{3}}{2}(S(n)+T(n)) \\
& \lambda_{3}=-\frac{1}{3} a_{2}(n)-\frac{1}{2}(S(n)+T(n))-\frac{i \sqrt{3}}{2}(S(n)+T(n))
\end{aligned}
$$

where $S(n)$ and $R(n)$ are numbers defined as

$$
S(n)=\sqrt[3]{R(n)+\sqrt{D(n)}}, \quad T(n)=\sqrt[3]{R(n)-\sqrt{D(n)}},
$$

such that $S(n)+T(n) \in \mathbb{R}$ and that $S(n)-T(n) \in \mathbb{R}$ for $D(n) \geq 0$, and $S(n)+T(n) \in \mathbb{R}$ and that $S(n)-T(n) \in$ $i \mathbb{R}$ for $D(n)<0$. In this particular case, we have for $n \neq 0$ that

$$
\begin{aligned}
& Q(n)=\frac{\gamma^{2}-6 \gamma+36}{9}=-\frac{(\gamma-3)^{2}+27}{9}<0 \\
& R(n)=\frac{-81 \gamma c^{2} n^{2}+(6+\gamma)\left(\gamma^{2}-15 \gamma+36\right)}{27} .
\end{aligned}
$$

Using this we see that $Q(n)=O(1)$ and $R(n)=O\left(n^{2}\right)$ for $|n|$ large enough, meaning that $D(n)=O\left(n^{4}\right)$ for $|n|$ large enough, and that $|S(n)|=|T(n)|=O\left(n^{2}\right)$ for $|n|$ large enough. Using previous facts and formulas (2.20)(2.22) for $|n|$ large enough, we conclude for $1 \leq m \leq 3$ and $|n|$ large enough

$$
\left|\lambda_{m}(n)\right| \simeq O\left(n^{2}\right),
$$

and so, for $|n|$ large enough and $1 \leq m \leq 6$, we have that

$$
\left|\beta_{m}(n)\right| \simeq O(|n|) .
$$

Using this fact it is not difficult to verify that in terms of the coefficient vectors $U^{\#}(n)$ we have the following equivalence of norms:

$$
\left\{\begin{array}{l}
\|U\|_{H}^{2} \sim \sum_{n \in \mathbb{Z}}\left(1+n^{2}\right)^{2}\left|U^{\#}(n)\right|^{2}, \\
\|U\|_{X}^{2} \sim \sum_{n \in \mathbb{Z}}\left(1+n^{2}\right)^{3}\left|U^{\#}(n)\right|^{2} .
\end{array}\right.
$$

From the equivalences in (2.25) it is evident that $\pi_{0}$ and $\pi_{1}$ are bounded on $H$ and on $X$ with $\pi_{0}+\pi_{1}=I$, and it is clear that $A X_{j} \subset H_{j}$ where $X_{j}=\pi_{j} X$ and $H_{j}=\pi_{j} H$ for $j=0,1$. This yields the spectral decompositions $H=H_{0} \oplus H_{1}$ and $X=X_{0} \oplus X_{1}$.

\subsection{Center manifolds of finite dimension and infinite codimension}

Here we consider an abstract differential equations of the form

$$
\frac{d u}{d y}(y)=A u(y)+f(u(y)) .
$$

where $X$ and $H$ are Banach spaces with $X$ densely embedded in $H, A \in \mathcal{L}(X, H)$, the space of bounded linear operators from $X$ to $H$, and $f$ is continuously differentiable from $H$ into $H$ with $f(0)=0$ and $D f(0)=0$. We will assume that the nonlinear part $f$ has the regularizing effect: $f(H) \subset X$. This hypothesis on $f$ is a stronger condition than those imposed in many works related with the existence center manifolds, but this completely natural for the Boussinesq type system considered in this paper. Existence of a local finite dimensional center manifold can be established in the same fashion as the approach used in Q uintero \& Pego (2002) in the case of having a center space with infinite dimension and infinite codimension for the Benney-Luke model. As in the later model, we must observe that the cutoff will be performed in the $H$ norm, and not in the $X$ norm as in Q uintero \& Pego (2002). This is important since we need to use an energy functional which is defined on $H$, which is conserved in time for classical solutions (taking values in $X$ ), but is indefinite in general. Fortunately for us, this energy controls the $H$ norm for solutions on the center manifold. So, we require obtaining a center manifold that contains solutions with large $X$ norm but small $H$ norm. This is a consequence that the nonlinearity $f$ has a smoothing property, mapping $H$ into $X$. 
We will state the result for the existence of a locally invariant center manifold of classical solutions for the system (2.26) under certain conditions which allow the center subspace (that associated with the purely imaginary spectrum of $A$ ) to have finite dimension and infinite codimension. We start with some basic definitions and some hypotheses:

Definition 2.1. Let $J \subset \mathbb{R}$ be an open interval and $u: \mathbb{R} \rightarrow$ $H$ be a function. We say that $u$ is a classical solution of (2.26) on $J$ if the mapping $y \mapsto u(y)$ is continuous from $J$ into $X$, is differentiable from $J$ into $H$ and (2.26) holds for all $y \in J$.

Let $\beta>0$, let $Y$ and $Z$ be Banach spaces and $U$ be an open set in $Y$. We define the Banach spaces $C_{b}(U, Z)$, $\operatorname{Lip}(U, Z)$ and $Y^{\beta}$ by

$$
\begin{gathered}
C_{b}(U, Z):=\left\{f \in C(U, Z): \sup _{u \in Z}\|f(u)\|_{Z}<\infty\right\} \\
\operatorname{Lip}(U, Z):=\left\{f \in C(U, Z):\|f(u)-f(v)\|_{Z}\right. \\
\left.\leq M_{f}\|u-v\|_{Y} \text { for all } u, v \in U\right\} \\
Y^{\beta}:=\left\{u \in C(\mathbb{R}, Y):\|u\|_{Y \beta}:=\sup _{t} e^{-\beta|t|}\|u(t)\|_{Y}<\infty\right\} .
\end{gathered}
$$

Throughout this section we assume that there are bounded projections $\pi_{0}$ and $\pi_{1}$ on $H$ such that

(i) (i) $H=H_{0} \oplus H_{1}$ with $H_{i}:=\pi_{i}(H)$,

(ii) $\left.\pi_{i}\right|_{X}$ is bounded from $X$ to $X$, and

(iii) $A X_{i} \subseteq H_{i}$ where $X_{i}:=\pi_{i}(X)$, for $i=0,1$.

We see that equation (2.26) can be rewritten as the first order system

$$
\begin{aligned}
& \frac{d}{d y} u_{0}(y)=A_{0} u_{0}(y)+\pi_{0} f(u(y)), \\
& \frac{d}{d y} u_{1}(y)=A_{1} u_{1}(y)+\pi_{1} f(u(y)),
\end{aligned}
$$

where $A_{i} \in \mathcal{L}\left(X_{i}, H_{i}\right)$ with $A_{i} y=\pi_{i} A y$ for $y \in X_{i}$.

We assume the following splitting properties for the operator $A_{1}$, associated with the linear evolution equation $d u / d y=A u$.

(H1) There exists $\alpha>0$ and a positive function $M_{1}$ on $[0, \alpha)$ such that for any $\beta \in[0, \alpha)$ and for any $g_{1} \in C\left(\mathbb{R}, X_{1}\right) \cap H_{1}^{\beta}$ the equation

$$
\frac{d}{d y} u_{1}=A_{1} u_{1}+g_{1}
$$

has a unique solution in $H_{1}^{\beta}$ given by $u_{1}=K_{1} g_{1}$, where $K_{1} \in \mathcal{L}\left(H_{1}^{\beta}\right)$ with $\left\|K_{1}\right\|_{\mathcal{L}\left(H_{1}^{\beta}\right)} \leq M_{1}(\beta)$. Furthermore $\left\|K_{1}\right\|_{\mathcal{L}\left(X_{1}^{\beta}\right)} \leq M_{1}(\beta)$.
As done by J. Q uintero and R. Pego in the case of the Benney-Luke model (see Q uintero \& Pego (2002)), we easily have that

Theorem 2.1 (Local Center Manifold Theorem). Let $H$, $X, A, \pi_{0}, \pi_{1}$ and $f$ be as above, and let

$$
B(\delta)=\left\{y \in H_{0}:\|y\|_{H}<\delta\right\} .
$$

Then for all sufficiently small $\delta>0$ there exists $\phi_{\delta}: H_{0} \rightarrow X_{1}$ such that

(i) $\phi_{\delta}(0)=0$ and $D \phi_{\delta}(0)=0$.

(ii) $\phi_{\delta} \in C_{b}\left(H_{0}, X_{1}\right) \cap \operatorname{Lip}\left(H_{0}, X_{1}\right)$, and on any ball $B\left(\delta^{\prime}\right)$, $\phi_{\delta}$ has Lipschitz constant $L\left(\delta^{\prime}\right)$ satisfying $L\left(\delta^{\prime}\right)<\frac{1}{2}$ and $L\left(\delta^{\prime}\right) \rightarrow 0$ as $\delta^{\prime} \rightarrow 0^{+}$.

(iii) The manifold $\mathcal{M}_{\delta} \subset X$ given by

$$
\mathcal{M}_{\delta}:=\left\{\xi+\phi_{\delta}(\xi): \xi \in X_{0}\right\}
$$

is a local integral manifold for (2.26) over $B(\delta) \cap X_{0}$. That is, given any $y \in \mathcal{M}_{\delta}$ there is a continuous map $u: \mathbb{R} \rightarrow \mathcal{M}_{\delta}$ with $u(0)=y$, such that for any open interval J containing 0 with $\pi_{0} u(J) \subset B(\delta)$ it follows that $u$ is a classical solution of (2.26) on J. Moreover, $u_{0}:=\pi_{0} u$ is the unique classical solution on J with $u_{0}(0)=\pi_{0} y$ to the reduced equation

$$
\frac{d}{d y} u_{0}(y)=A_{0} u_{0}(y)+F_{\delta}\left(u_{0}(y)\right)
$$

where $F_{\delta}: H_{0} \rightarrow X_{0}$ is locally Lipschitz and is given by $F_{\delta}(w):=\pi_{0} f\left(w+\phi_{\delta}(w)\right)$.

(iv) For any open interval $J \subset \mathbb{R}$, every classical solution $u_{0} \in C^{1}\left(J, H_{0}\right) \cap C\left(J, X_{0}\right)$ of the reduced equation (2.30) such that $u_{0}(y) \in B(\delta)$ for all $y \in J$ yields, via $u=u_{0}+\phi_{\delta}\left(u_{0}\right)$, a classical solution $u$ of the full equation (2.26) on J.

(v) The manifold $\mathcal{M}_{\delta}$ contains all classical solutions on $\mathbb{R}$ that satisfy $\|u(y)\|_{H} \leq \delta$ for all $y$.

\subsection{Linear dynamics analysis.}

Now we are interested in establishing the solvability conditions to the linear level. First we consider the center subspace $H_{0}$. We define the bounded $C_{0}$-group $\left\{S_{0}(y)\right\}_{y \in \mathbb{R}}$ on $H_{0}$ with infinitesimal generator $\mathcal{A}_{0}=$ $\left.\mathcal{A}\right|_{X_{0}}$ by

$$
S_{0}(y) U=\sum_{0 \leq|n| \leq n_{0}} \sum_{m=1}^{2} v_{m}(n) U_{m}^{\#}(n) e^{\beta_{m}(n) y} e^{i n x} .
$$


Now, we want to determine the Green function to solve the problem in the hyperbolic subspace $H_{1}$. Let us consider the inhomogeneous linear equation

$$
\frac{d}{d y} U(t)=\mathcal{A}_{1} U(y)+G(y)
$$

where $\mathcal{A}_{1}=\left.\mathcal{A}\right|_{X_{1}}$. We need to observe that $\mid \Re\left(\lambda_{m}(n) \mid \geq\right.$ $\alpha>0$ for all $n \in \mathbb{Z}$, except for $m=1,2$ when $n \neq 0$. Let $0 \leq \varrho<\alpha$ and let $G \in C\left(\mathbb{R}, X_{1}\right) \cap H_{1}^{\varrho}$, where for any Banach space $Y$,

$$
Y^{\varrho}=\left\{u \in C(\mathbb{R}, Y) \mid\|u\|_{Y e}:=\sup _{y} e^{-\varrho|y|}\|u\|_{Y}<\infty\right\} .
$$

Hereafter we will assume $m$ and $n$ are such that $1 \leq m \leq$ 6 and $n \in \mathbb{Z} \backslash\{0\}$, or $m=5,6$ for $n=0$. Suppose $U \in C^{1}\left(\mathbb{R}, H_{1}\right) \cap C\left(\mathbb{R}, X_{1}\right)$ is a solution belonging to $H_{1}^{\rho}$. Then using the Fourier series expansion in $x$ and multiplying by the matrix $Z(n)$ yields the differential equation

$$
\frac{d}{d y} U_{m}^{\#}(n, y)=\beta_{m}(n) U_{m}^{\#}(n, y)+G_{m}^{\#}(n, y)
$$

The functions $G_{m}^{\#}(n, \cdot)$ and $U_{m}^{\#}(n, \cdot)$ belong to $\mathbb{R}^{\varrho}(Y=\mathbb{R}$ in (2.33)). From the fact that $\left|\beta_{m}(n)\right| \geq \alpha>\varrho$, we conclude necessarily that

$$
\left\{\begin{array}{l}
U_{u}^{\#(n, y)}=\int_{\infty}^{y} e^{\beta_{u}(k)(y-\tau)} G_{u}^{\#}(n, \tau) d \tau, \\
U_{s}^{\#}(n, y)=\int_{-\infty}^{y} e^{\beta_{s}(n)(y-\tau)} G_{s}^{\#}(n, \tau) d \tau .
\end{array}\right.
$$

where for $0<|n| \leq n_{0}$ we have $u=3,5$ and $s=4,6$, and for $|n|>n_{0}$ we have $u=1,3,5$ and $s=2,4,6$. As a direct consequence any solution of (2.32) in $H_{1}^{o}$ is unique. We will see that the formulas (2.35) together with the representation for $U=\pi_{1} U$ in (2.19) allow us to establish the existence of a solution in $H_{\varrho}^{1}$. To see this, we decompose equation (2.32) using projections into the "unstable" and "stable" subspaces. The projections for $U \in H$ are

$$
\begin{aligned}
& \pi_{u} U=\sum_{0 \leq|n| \leq n_{0}} \sum_{m=3,5} v_{m}(n) U_{m}^{\#}(n) e^{i n x} \\
&+\sum_{|n|>n_{0}} \sum_{m=1,3,5} v_{m}(n) U_{m}^{\#}(n) e^{i n x} . \\
& \pi_{s} U=\sum_{0 \leq|n| \leq n_{0}} \sum_{m=4,6} v_{m}(n) U_{m}^{\#}(n) e^{i n x} \\
& \quad+\sum_{|n|>n_{0}} \sum_{m=2,4,6} v_{m}(n) U_{m}^{\#}(n) e^{i n x} .
\end{aligned}
$$

Clearly $\pi_{u}$ and $\pi_{s}$ are bounded on $H$ and $X$ and $\pi_{u}+$ $\pi_{s}=\pi_{1}$. Now, we introduce a Green's function operator
$S(y)$ defined for nonzero $y \in \mathbb{R}$ by

$$
S(y) U=\left\{\begin{array}{l}
-\sum_{0 \leq|n| \leq n_{0} m=3,5} \sum_{m} v_{m}(n) U_{m}^{\#}(n) e^{\beta_{m}(n) y} e^{i n x} \\
-\sum_{|n|>n_{0} m=1,3,5} v_{m}(n) U_{m}^{\#}(n) e^{\beta_{m}(n) y} e^{i n x}, y<0, \\
\sum_{0 \leq|n| \leq n_{0} m=4,6} v_{m}(n) U_{m}^{\#}(n) e^{\beta_{m}(n) y} e^{i n x} \\
+\sum_{|n|>n_{0} m=2,4,6} v_{m}(n) U_{m}^{\#}(n) e^{\beta_{m}(n) y} e^{i n x}, y>0 .
\end{array}\right.
$$

By the definition of the norm in $H$ and $X$ (see (2.25)), we have that

$$
\begin{aligned}
\left\|\pi_{1} U\right\|_{X}^{2}= & \sum_{0 \leq|n| \leq n_{0}} \sum_{m=3}^{6}\left|U_{m}^{\#}(n)\right|^{2} \\
& +\left.\left.\sum_{|n|>n_{0}} \sum_{m=1}^{6}\left(1+n^{2}\right)\right|^{3} U_{m}^{\#}(n)\right|^{2}, \\
\left\|\pi_{1} U\right\|_{H}^{2}=\sum_{0 \leq|n| \leq n_{0}} \sum_{m=3}^{6}\left|U_{m}^{\#}(n)\right|^{2} & \\
& +\left.\left.\sum_{|n|>n_{0}} \sum_{m=1}^{6}\left(1+n^{2}\right)\right|^{3} U_{m}^{\#}(n)\right|^{3} .
\end{aligned}
$$

On the other hand, for $1 \leq m \leq 6$ and $y \neq 0$ and $n \neq 0$, we have that

$$
\left|e^{\beta_{m}(n) y}\right|=e^{\Re\left(\beta_{m}(n)\right) y} \leq e^{-\alpha|y|} .
$$

Using this fact, we see directly for $Y=H$ or $X$ that,

$$
\begin{aligned}
& \|S(y) U\|_{Y} \leq\left(\left|U_{5}^{\#(0)}\right|^{2}+\left|U_{6}^{\#(0)}\right|^{2}\right) e^{\Re\left(\beta_{5}(0)\right) y} \\
& +\sum_{n \in \mathbb{Z} \backslash\{0\}} \sum_{l}\left|U_{l}^{\#}(n)\right|^{2} e^{-2 \Re\left(\beta_{l}(n)\right) y} \\
& \leq e^{-2 x|y|}\|U\|_{Y}^{2} .
\end{aligned}
$$

We also have for $y>0$ that

$$
\begin{gathered}
\sup _{\lambda \geq \alpha} \lambda^{-1}\left|e^{-\lambda y}-1\right| \leq t, \\
\sup _{\lambda \geq \alpha} \lambda e^{-\lambda y}= \begin{cases}\alpha e^{-\alpha y}, & \alpha y \geq 1, \\
1 / e y, & \alpha y \leq 1 .\end{cases}
\end{gathered}
$$

Following the same type of calculation and using previous facts, one can easily verify that for some constant $C$ (independent of $y$ ) we have the following norm bounds:

$$
\begin{gathered}
\|S(y)\|_{\mathcal{L}(Y) \leq C e^{-\alpha|y|} \quad(Y=H \text { or } X)} \\
\|S(y)\|_{\mathcal{L}(H, X)} \leq\left\{\begin{array}{l}
C e^{-\alpha y}, \alpha|t| \geq 1 \\
C|y|^{-1}, \alpha|y| \leq 1
\end{array}\right.
\end{gathered}
$$




$$
\left\|S(y)-\pi_{S}\right\|_{\mathcal{L}(X, H)}+\left\|S(-y)+\pi_{u}\right\|_{\mathcal{L}(X, H)} \leq C y, \quad y>0,
$$

where $\mathcal{L}(Y)$ and $\mathcal{L}(H, X)$ respectively denote the space of bounded operators on $Y$, and from $H$ to $X$. Clearly, we have that $S(y) \rightarrow \pi_{s}$ (resp. $-\pi_{u}$ ) strongly as $y \rightarrow$ $0^{+}$(resp. $0^{-}$). Therefore the families $\{S(y)\}_{y>0}$ and $\{-S(-y)\}_{y>0}$ are analytic semigroups in $\pi_{S} H$ and $\pi_{u} H$ respectively (?, p.62). Moreover, we also have that $S$ is $C^{1}$ from $\mathbb{R} \backslash\{0\}$ to $\mathcal{L}(H)$ with $d S(y) / d y=A_{1} S(y)$. There fore, we conclude that equation (2.35) yield the formula

$$
U(y)=\int_{-\infty}^{\infty} S(y-\tau) G(\tau) d \tau
$$

for the solution of (2.32). Finally, we need to establish that $U \in C(\mathbb{R}, X), U \in H_{1}^{e}$, and $d U / d y$ exists in $H$ and satisfies (2.32). The computations in this case follows as those done for the Benney-Luke equation in ?, but for completeness we include the details. First observe that

$U(y)=\int_{|s| \leq \alpha^{-1}} S(s) G(y-s) d s+\int_{|s| \geq \alpha^{-1}} S(s) G(y-s) d s$.

The first term is in $C(\mathbb{R}, X)$ since $G \in C(\mathbb{R}, X)$ and we have the estimate (2.39). Now, since $G \in H^{e}$, the we may use (2.40) to see that the second integral converges in $X$ uniformly on compact sets in $y$. Then we have that $U \in C(\mathbb{R}, X)$.

On the other hand, using (2.39) we have for $Y=H$ or $X$ that $U \in Y^{\varrho}$. In fact, if $G \in Y^{\varrho}$ then

$$
\begin{aligned}
e^{-\varrho|y|}\|U(y)\|_{Y} & \leq C\|G\|_{Y} \int_{-\infty}^{\infty} e^{-\alpha|s|+\varrho(|y-s|-|y|)} d s \\
& \leq C\|G\|_{Y} \int_{-\infty}^{\infty} e^{-(\alpha-\varrho)|s|} d s \\
& \leq \frac{2 C}{\alpha-\varrho}\|G\|_{Y} \rho
\end{aligned}
$$

Moreover, we have that

$$
\|U(y)\|_{Y e} \leq \frac{2 C}{\alpha-\varrho}\|G\|_{Y e} .
$$

It remains to show $U=\pi_{s} U+\pi_{u} U$ is differentiablein $H$ and satisfies (2.32). We check in a standard fashion that $\pi_{s} U$ is differentiable. For $h>0$ we compute

$$
\begin{array}{r}
\frac{\pi_{s} U(y+h)-\pi_{s} U(y)}{h}=\left(\frac{S(h)-\pi_{s}}{h}\right) \pi_{s} U(y) \\
+\frac{1}{h} \int_{0}^{h}\left(S(\tau)-\pi_{s}\right) G(t+h-\tau) d \tau \\
+\frac{1}{h} \int_{y}^{y+h} \pi_{s} G(\tau) d \tau .
\end{array}
$$

Using (2.41) and $G \in C(\mathbb{R}, X)$, as $h \rightarrow 0^{+}$we deduce that

$$
\begin{aligned}
\lim _{h \rightarrow 0^{+}}\left(\frac{S(h)-\pi_{s}}{h}\right) \pi_{s} U(y) & =\mathcal{A}_{1} \pi_{s} U(y), \\
\lim _{h \rightarrow 0^{+}} \frac{1}{h} \int_{y}^{y+h} \pi_{s} G(\tau) d \tau & =\pi_{s} G(y) .
\end{aligned}
$$

Since we have that $S(y) \rightarrow \pi_{s}$ strongly as $y \rightarrow 0^{+}$, the we have that

$$
\lim _{h \rightarrow 0^{+}} \frac{1}{h} \int_{0}^{h}\left(S(\tau)-\pi_{s}\right) G(t+h-\tau) d \tau=0 .
$$

Hence the right derivative exists and satisfies $D_{+} \pi_{s} U(t)=A_{1} \pi_{s} U(t)+\pi_{s} G(t)$, so is continuous into $H$. It follows that $\pi_{s} U$ is differentiable. We may treat $\pi_{u} U$ in a similar way, and conclude that $U$ is differentiable and satisfies (2.32). So, from the Theorem 2.1, we have established that system (2.8) admits a local center manifold having the properties stated in the Theorem.

\subsection{G lobal existence and stability for $\gamma>0$ and $|c|>1$ (large enough)}

Weare now interested in proving global existence of classical solutions on the local center manifold, for initial data that is small in $H$-norm, which follows from the fact that the zero solution is stable on the center manifold characterized by the graph of a function $\phi_{\delta}: H_{0} \rightarrow X_{1}$. We use strongly the existence an energy functional that is conserved in time for classical solutions. We define the energy functional $\mathcal{E}: H \rightarrow \mathbb{R}$ by $\mathcal{E}(U)=\mathcal{E}_{0}(U)+\mathcal{E}_{1}(U)$, where the quadratic part is

$$
\begin{aligned}
\mathcal{E}_{0}(U)= & \frac{1}{2 \pi} \int_{0}^{2 \pi}\left(-\left|u_{1}\right|^{2}-\frac{1}{6}\left|\partial_{x} u_{1}\right|^{2}+\left|u_{2}\right|^{2}+\right. \\
& \frac{1}{3}\left|\partial_{x} u_{2}\right|^{2}+\frac{1}{6}\left|u_{3}\right|^{2}-\left|u_{5}\right|^{2}-\frac{1}{\gamma}\left|\partial_{x} u_{5}\right|^{2} \\
& \left.\frac{1}{\gamma}\left|u_{6}\right|^{2}+2 c u_{5} u_{1}\right) d x-\frac{1}{6 \pi}\left(u_{4}, u_{2}\right)_{-1,1},
\end{aligned}
$$

where $(\cdot, \cdot)_{-1,1}$ represents the pairing between $\tilde{H}^{-1}$ and $\tilde{H}^{1}$, and the remaining part is

$$
\mathcal{E}_{1}(U)=\frac{1}{\pi(p+1)} \int_{0}^{2 \pi} u_{5}\left(p u_{2}^{p+2}-u_{1}^{p+2}\right) d x .
$$

From the definition, $\mathcal{E}$ is a smooth function from $H$ to $\mathbb{R}$. After multiplying appropriately the equation (2.6) by $u_{2}$ and equation (2.7) by $u_{6}$, one can easily verify that if $U \in C^{1}(\mathbb{R}, H)$ is a classical solution of the first order equation (2.8), then for all $y \in \mathbb{R}$

$$
\frac{d}{d y} \mathcal{E}(U(y))=0
$$


Even though previous property, this energy can not be used to obtain a solution throughout the variational method since neither $\mathcal{E}$ nor $\mathcal{E}_{0}$ is not positive define in the space $H$. We will see that energy $\mathcal{E}_{0}$ is positive on the center space $H_{0}$, and also that this controls the norm of $U$ in $H$, via the center manifold result. We note that from the definition of the variable $U=\left(u_{1}, u_{2}, u_{3}, u_{4}, u_{5}, u_{6}\right)$, we have a priori that $u_{1}=\partial_{x} v$ has mean zero on $[0,2 \pi]$, meaning that $\widehat{U}_{1}(0)=0$.

Lemma 2.1. Let $|c|>1$ large enough and $\gamma>0$. Then there is a positive constant $M_{0}>1$ such that for any $U \in H_{0}$ with $\widehat{U}_{1}(0)=0$,

$$
M_{0}^{-1}\|U\|_{H}^{2} \leq \mathcal{E}_{0}(U) \leq M_{0}\|U\|_{H}^{2} .
$$

Proof. From the Fourier series representation of $U \in H_{0}$ given with $\widehat{U}_{1}(0)=0$, we have that

$$
\begin{aligned}
& U=\left(\begin{array}{c}
0 \\
U_{2}^{\#}(0) \\
0 \\
0 \\
0 \\
0
\end{array}\right) \\
&+\sum_{0 \leq|n| \leq n_{0}}\left(\begin{array}{c}
i n\left(U_{1}^{\#}(n)+U_{2}^{\#}(n)\right) \\
\beta_{1}(n)\left(U_{1}^{\#}(n)-U_{2}^{\#}(n)\right) \\
\beta_{1}^{2}(n)\left(U_{1}^{\#}(n)+U_{2}^{\#}(n)\right) \\
\beta_{1}^{3}(n)\left(U_{1}^{\#}(n)-U_{2}^{\#}(n)\right) \\
-\frac{c n \gamma i}{\Theta_{1}(n)}\left(U_{1}^{\#}(n)+U_{2}^{\#}(n)\right) \\
-\frac{c n \gamma \beta_{1}(n) i}{\Theta_{1}(n)}\left(U_{1}^{\#}(n)-U_{2}^{\#}(n)\right)
\end{array}\right) e^{i n x} \\
&=\widehat{U}(0)+\sum_{0 \leq|n| \leq n_{0}} \widehat{U}(n) e^{i n x} .
\end{aligned}
$$

We also have that $\mathcal{E}_{0}(U)=\sum_{0 \leq|n| \leq n_{0}} \mathcal{E}_{0}\left(\widehat{U}(n) e^{i n x}\right)$. We note that

$$
\mathcal{E}_{0}(\hat{U}(0))=\left|U_{2}^{\#}(0)\right|^{2},
$$

On the other hand, for $\beta_{1}(n)=\beta$ we have that

$$
\begin{aligned}
\mathcal{E}_{0}\left(\widehat{U}(n) e^{i n x}\right)=\Gamma_{1}(n)\left|U_{1}^{\#}(n)+U_{2}^{\#}(n)\right|^{2} & \\
& +\Gamma_{2}(n)\left|U_{1}^{\#}(n)-U_{2}^{\#}(n)\right|^{2}
\end{aligned}
$$

where

$$
\begin{aligned}
& \Gamma_{1}(n)=-n^{2}-\frac{n^{4}}{6}+\frac{\beta^{4}}{6}-\frac{c^{2} n^{2} \gamma}{\Theta_{1}^{2}(n)}\left(n^{2}+\gamma\right)-\frac{2 c^{2} n^{2} \gamma}{\Theta_{1}(n)} \\
& \Gamma_{2}(n)=|\beta|^{2}\left(1+\frac{n^{2}}{3}+\frac{c^{2} n^{2} \gamma}{\Theta_{1}^{2}(n)}\left(n^{2}+\gamma\right)-\frac{\beta^{2}}{3}\right)
\end{aligned}
$$

Since for $|c|>1$ (large enough) we have that $\beta_{1}^{2}(n)=$ $\lambda_{1}(n)<0$ for $0<n \leq n_{0}$, then we have the right side of the second term is positive. Now, for the first term, we use the polynomial equation for $\beta_{1}(n)$ given in (2.11). First note that

$$
-n^{2}-\frac{n^{4}}{6}+\frac{\beta^{4}}{6}-\frac{c^{2} n^{2} \gamma}{\Theta_{1}^{2}(n)}\left(n^{2}+\gamma\right)-\frac{2 c^{2} n^{2} \gamma}{\Theta_{1}(n)}=\frac{L_{1}+L_{2}}{6 \Theta_{1}^{2}(n)}
$$

where $L_{1}$ and $L_{2}$ are given by

$$
\begin{aligned}
& L_{1}=\left(\beta^{4}-n^{2}\left(n^{2}+6\right)\right) \Theta_{1}^{2}(n), \\
& L_{2}=-12 c^{2} n^{2} \gamma \Theta_{1}(n)-6 c^{2} n^{2} \gamma\left(n^{2}+\gamma\right) .
\end{aligned}
$$

But we have that

$$
\begin{aligned}
L_{1} & =\beta^{8}-2\left(n^{2}+\gamma\right) \beta^{6}+\left[\left(n^{2}+\gamma\right)^{2}-n^{2}\left(n^{2}+6\right)\right] \beta^{4} \\
& +2 n^{2}\left(n^{2}+\gamma\right)\left(n^{2}+6\right) \beta^{2}-n^{2}\left(n^{2}+\gamma\right)^{2}\left(n^{2}+6\right) \\
L_{2} & =-12 c^{2} n^{2} \gamma \beta^{2}+6 c^{2} n^{2}\left(n^{2}+\gamma\right) .
\end{aligned}
$$

Using this, we get that

$$
\begin{aligned}
L_{1}+ & L_{2}=\beta^{8}-2\left(n^{2}+\gamma\right) \beta^{6} \\
& +\left[\left(n^{2}+\gamma\right)^{2}-n^{2}\left(n^{2}+6\right)\right] \beta^{4} \\
& +\left(2 \beta^{2}-\left(n^{2}+\gamma\right)\right)\left[\left(n^{2}+\gamma\right)\left(n^{2}+6\right)-6 c^{2} n^{2}\right] n^{2} .
\end{aligned}
$$

But from (2.11), we have that

$$
\begin{aligned}
\beta^{6}- & \left(6+3 n^{2}+\gamma\right) \beta^{4} \\
& +\left[\left(6+2 n^{2}\right)\left(n^{2}+\gamma\right)+n^{2}\left(6+n^{2}\right)\right] \beta^{2} \\
& =n^{2}\left[\left(6+n^{2}\right)\left(n^{2}+\gamma\right)-6 \gamma c^{2}\right] .
\end{aligned}
$$

Then using that $\beta_{1}^{2}(n)<0$ for $0<n \leq n_{0}$, we finally get that

$$
\begin{aligned}
L_{1}+L_{2}=3 \beta^{8}- & {\left[2\left(6+3 n^{2}+\gamma\right)\right.} \\
+ & \left.3\left(n^{2}+\gamma\right)\right] \beta^{6}+ \\
{\left[2\left(n^{2}+\gamma\right)(\right.} & \left.\left.4 n^{2}+\gamma+9\right)+n^{2}\left(n^{2}+6\right)\right] \beta^{4} \\
- & \left(n^{2}+\gamma\right)\left(n^{4}+2 n^{2}+6 \gamma\right) \beta^{2} \geq 0 .
\end{aligned}
$$

This fact implies that

$$
\begin{aligned}
& \min _{0<n \leq n_{0}}\left(\Gamma_{1}(n), \Gamma_{2}(n)\right)\left(\left|U_{1}^{\#}(n)+U_{2}^{\#}(n)\right|^{2}\right. \\
&\left.+\left|U_{1}^{\#}(n)-U_{2}^{\#}(n)\right|^{2}\right) \leq \mathcal{E}_{0}\left(\widehat{U}(n) e^{i n x}\right) \\
& \leq \max _{0<n \leq n_{0}}\left(\Gamma_{1}(n), \Gamma_{2}(n)\right)\left(\left|U_{1}^{\#}(n)+U_{2}^{\#}(n)\right|^{2}\right. \\
&\left.+\left|U_{1}^{\#}(n)-U_{2}^{\#}(n)\right|^{2}\right),
\end{aligned}
$$

which implies that

$$
\begin{aligned}
\min _{0 \leq n \leq n_{0}}\left(\Gamma_{1}(n), \Gamma_{2}(n)\right)\left(\left|U_{1}^{\#}(n)\right|^{2}\right. & \\
& \left.+\left|U_{2}^{\#}(n)\right|^{2}\right) \leq \mathcal{E}_{0}\left(\widehat{U}(n) e^{i n x}\right) \\
\leq & \max _{0 \leq n \leq n_{0}}\left(\Gamma_{1}(n), \Gamma_{2}(n)\right)\left(\left|U_{1}^{\#}(n)\right|^{2}+\left|U_{2}^{\#}(n)\right|^{2}\right),
\end{aligned}
$$


In other words, we have shown that

$$
\mathcal{E}(U) \sim \sum_{0 \leq n \leq n_{0}}\left(1+n^{2}\right)^{2}\left(\left|U_{1}^{\#}(n)\right|^{2}+\left|U_{2}^{\#}(n)\right|^{2}\right) \sim\|U\|_{H}^{2} .
$$

The first consequence of this fact is the following result:

Corollary 2.1. Let $|c|>1$ large enough and $\gamma>0$. Then there are $\delta_{1}>0$ and $M_{1}>1$ such that for any $U \in H_{0}$ with $\widehat{U}_{1}(0)=0$ and $\|U\|_{H}<\delta_{1}$,

$$
\frac{1}{M_{1}}\|U\|_{H}^{2} \leq|\mathcal{E}(U)| \leq M_{1}\|U\|_{H}^{2} .
$$

Proof. First note that from the Hölder inequality with $q=p+2$, there is some positive constant $C=C(p)$ (independent of $U$ ) such that

$$
\begin{aligned}
\left|\mathcal{E}_{1}(U)\right| & =\left|\frac{1}{\pi(p+1)} \int_{0}^{2 \pi} u_{5}\left(p u_{2}^{p+2}-u_{1}^{p+2}\right) d x\right| \\
& \leq C\left\|u_{5}\right\|_{\tilde{H}^{1}}\left(\left\|u_{2}\right\|_{\tilde{H}^{1}}^{p+1}+\left\|u_{1}\right\|_{\tilde{H}^{1}}^{p+1}\right) \\
& \leq C\|U\|_{H}^{p+2}
\end{aligned}
$$

Moreover for some constants $C_{0}$ and $C_{1}=C_{1}(p)$ (independent of $U$ ), we conclude that

$$
\begin{aligned}
|\mathcal{E}(U)| & \geq\left|\mathcal{E}_{0}(U)\right|-\left|\mathcal{E}_{1}(U)\right| \\
& \geq C_{0}\|U\|_{H}^{2}-C_{1}\|U\|_{H}^{p+2} \\
& \geq\|U\|_{H}^{2}\left(C_{0}-C_{1}\|U\|_{H}^{p}\right) .
\end{aligned}
$$

Let $\delta_{1}>0$ be such that $C_{0}-\delta_{1}^{p} C_{1}>0$. Then for $\|U\|_{H} \leq \delta_{1}$ with $U \in H_{0} \backslash\{0\}$ we have that

$$
|\mathcal{E}(U)| \geq\|U\|_{H}^{2}\left(C_{0}-C_{1} \delta_{1}^{p}\right) .
$$

The second claim of this lemma follows directly. In fact, for $U \in H$ we have that

$$
\begin{aligned}
|\mathcal{E}(U)| & \leq C\left(\|U\|_{H}^{2}+\|U\|_{H}^{p+2}\right) \\
& \leq C\|U\|_{H}^{2}\left(1+\|U\|_{H}^{p}\right) .
\end{aligned}
$$

Now, we are interested in estimating the energy $\mathcal{E}$ on the center manifold. In other words, we want to obtain a similar estimates for the lift of $\mathcal{E}$ to the center manifold $\mathcal{M}_{\delta}$.

Lemma 2.2. $s l$ Let $\phi_{\delta}$ as in Theorem 2.1. Then there exist constants $\delta_{2}>0$ and $C_{2}>1$ such that for all $\xi \in H_{0}$ with $\|\xi\|_{H}<\delta_{2}$ we have

$$
\frac{1}{C_{2}}\|\xi\|_{H}^{2} \leq \mathcal{E}\left(\xi+\phi_{\delta}(\xi)\right) \leq C_{2}\|\xi\|_{H}^{2} .
$$

Proof. Let us define the functional $\tilde{\mathcal{E}}: H_{0} \rightarrow \mathbb{R}$ by

$$
\tilde{\mathcal{E}}(\xi):=\mathcal{E}\left(\xi+\phi_{\delta}(\xi)\right),
$$

where the function $\phi_{\delta}$ is defined in Theorem (2.1). First note that $\left\|\phi_{\delta}(\xi)\right\|_{H}=o\left(\|\xi\|_{H}\right)$. Since $\mathcal{E}$ is smooth and $\mathcal{E}(0)=0$, then $\mathcal{E}^{\prime}(\xi)=O\left(\|\xi\|_{H}\right)$. As a consequence of this fact and that $\mathcal{E}_{1}(U)=O\left(\|U\|_{H}^{p+2}\right)$, we have for $\xi \in H_{0}$ that

$$
\begin{aligned}
\mathcal{E}\left(\xi+\phi_{\delta}(\xi)\right)=\mathcal{E}_{0}(\xi) & \\
+O\left(\|\xi\|_{H}\left\|\phi_{\delta}(\xi)\right\|_{H}\right)+ & \mathcal{E}_{1}\left(\xi+\phi_{\delta}(\xi)\right) \\
& =\mathcal{E}_{0}(\xi)+o\left(\|\xi\|_{H}^{2}\right) .
\end{aligned}
$$

as $\|\xi\|_{H} \rightarrow 0$. Then by the previous result, we get the conclusion.

We first establish that solutions starting in the center manifold are appropriately bounded.

Lemma 2.3. Let $\xi \in X_{0}$ be such that $\widehat{\xi}_{1}(0)=0$ and that $\|\xi\|_{X} \leq \delta_{2}$. There exists a unique classical solution $U(\xi, \cdot)$ for the full problem (without cutoff) (2.8) on $\mathbb{R}$ with initial condition $\pi_{0} \circ U(\xi, 0)=\xi$ such that on any open interval $J$ containing 0 ,

$$
\left\|U_{0}(\xi, y)\right\|_{H} \leq C_{2}\|\xi\|_{H} \text { for any } y \in J .
$$

Proof. We may assume that $\delta_{2}$ small enough such that $\delta_{2}<<\delta$. Let $\xi \in X_{0}$ be such that $\|\xi\|_{X} \leq \delta_{2}$. Now, from Theorem 2.1, there exists a unique continuous function $U$ form $\mathbb{R}$ to the local center manifold $\mathcal{M}_{\delta}$ such that $\pi_{0}(U(0))=\xi$, which turns out to be a classical solution of the equation (2.8) on any open interval $J \subset \mathbb{R}$ containing 0 such that $\left\|\pi_{0}(U(y))\right\|_{H} \leq \delta$ for any $y \in J$. On the other hand, since $U$ is a classical solution and the energy $\mathcal{E}$ is conserved, then we have for any $y \in J$ that

$$
\frac{1}{C_{2}}\left\|\pi_{0}(U(y))\right\|_{H}^{2} \leq \mathcal{E}\left(\pi_{0}(U(y))\right)=\mathcal{E}(U(0)) \leq C_{2}\|\xi\|_{H}^{2},
$$

meaning $\left\|\pi_{0}(U(y))\right\|_{H} \leq C_{2}\|\xi\|_{H}$, for any $y \in J$ as desired. A continuation argument shows that $U$ is a classical solution for the full problem (without cutoff) (2.8) on $\mathbb{R}$.

Now we are in position to state the main result on the existence and the stability on the center manifold. The proof of this result follows in the same fashion as the Benney-Luke equation done by $\mathbf{Q}$ uintero and Pego in ?.

Theorem 2.2. (G lobal Existence and stability on the center manifold) Let $\phi_{\delta}$ be given by applying Theorem 2.1 to (2.8). There exist positive constants $\delta_{3}$ and $C_{3}$ such that, for any $\xi \in X_{0}$ with $\widehat{\xi}_{1}(0)=0$ and $\|\xi\|_{H} \leq \delta_{3}$, there is a unique classical solution $U$ on $\mathbb{R}$ to (2.8) such that $\pi_{0} U(0)=\xi$ and $\|U(y)\|_{H} \leq 2 C_{2}\|\xi\|_{H}$ for all $y \in \mathbb{R}$. Moreover, for any $T>0$ the map taking $\xi$ to $U$ is Lipschitz continuous from $\mathrm{H}_{0}$ to $C([-T, T], H)$. 
Acknowledgments. J. R. Q uintero was supported by the Mathematics Department at Universidad del Valle (Colombia) under the project C.I. 7910.

\section{References}

G rover, M . (2001). An existence theory for three-dimensional periodic travelling gravity-capillary water waves with bounded transverse profiles. Physica D 152(1): 295-415.

G rover, M., Mielke, A. (2001). A spatial dynamics approach to three-dimensional gravity-capillary steady water waves. Proc. Royal Soc. Edin. A131(1): 83-136.

Grover, M., Schneider, A. (2001). Modulating pulse solutions for a class of nonlinear wave equations. Commun. Math. Phys. 219: 489-522.

H aragus-Courcelle M ., Schneider G . (1999). Bifurcating fronts for the Taylor-Couette problem in infinite cylinders. Z. Angew. Math. Phys. 50: 120-151.

Kirchgässner, A. (1982). Wave solutions of reversible systems and applications. J. Diff. Eqns. 45: 113-127.

Mielke, A. (1988). Reduction of quasilinear elliptic equations in cylindrical domains with applications. Math. Meth. Appli. Sci. 101: 51-56.

Mielke, A. (1991). Hamiltonian and Lagrangian Flows on Center Manifolds. Berlin: Springer-Verlag.
Mielke, A. (1992). On nonlinear problems of mixed type: a qualitative theory using infinite-dimensional center manifolds. J. Dyn. Diff. Eqns. 4 (1): 419-443.

Pazy, A . (1983). Semigroups of Linear Operators and Applications to Partial Differential Equations. Springer-Verlag, New York.

Q uintero J., Pego R. (1999). Two-dimensional solitary waves for a Benney-Luke equation. Physica D 132: 476-496.

Q uintero J., Pego R. (2002). A host of Travelling Waves in a Model of Three-Dimensional Water-Wave Dynamics. Nonlinear Science 12: 59-83.

Sandstede B., Scheel A. (1999). An Essential instability of pulses and bifurcations to modulated travelling waves. Proc. Roy. Soc. Edin. A 129: 1263-1290.

Sandstede B., Scheel A. (2004). Defects in oscillatory media: Toward a classification. SIAM J. Appl. Dynam. Syst. 3: 1-68.

Scarpellini, B. (1990). On nonlinear problems of mixed type: a qualitative theory using infinite-dimensional center manifold. I and II. J. Appl. Math. Phys. (ZAMP). 42: 289-314.

Vanderbauwhede, A., and looss, G. (1982). On nonlinear problems of mixed type: a qualitative theory using infinitedimensional center manifold. I and II. Dynamics Reported, New Series 1: 125-163.

Vanderbauwhede A . (1989). Centre manifolds, normal forms and elementary bifurcations. Dynamics Reported, New Series 2: 89-169. 